\title{
A puzzling solar cell structure: an exercise to get insight on intermediate band solar cells
}

\author{
A.Martí , E. Antolín ，P.García-Linares ， I. Ramiro , E. López ， I. Tobías and A.Luque
}

\begin{abstract}
We introduce one trivial but puzzling solar cell structure. It consists of a high bandgap pn junction (top cell) grown on a substrate of lower bandgap. Let us assume, for example, that the bandgap of the top cell is $1.85 \mathrm{eV}\left(\mathrm{Al}_{0.3} \mathbf{G a}_{0.7} \mathrm{As}\right)$ and the bandgap of the substrate is $1.42 \mathrm{eV}$ (GaAs). Is the opencircuit of the top cell limited to $1.42 \mathrm{~V}$ or to $1.85 \mathrm{~V}$ ? If the answer is " $1.85 \mathrm{~V}$ " we could then make the mind experiment in which we illuminate the cell with $1.5 \mathrm{eV}$ photons (notice these photons would only be absorbed in the substrate). If we admit that these photons can generate photocurrent, then because we have also admitted that the voltage is limited to $1.85 \mathrm{~V}$, it might be possible that the electron-hole pairs generated by these photons were extracted at $1.6 \mathrm{~V}$ for example. However, if we do so, the principles of thermodynamics could be violated because we would be extracting more energy from the photon than the energy it initially had. How can we then solve this puzzle?
\end{abstract}

Index Terms - intermediate band solar cell, quantum efficiency model, solar cell modeling.

\section{INTRODUCTION}

Fig. 1 shows the structure of a high bandgap solar cell (that we will designate as top cell) grown on top of a substrate of lower bandgap. Usually, the substrate is considered photovoltaic inactive but here we will not make this assumption since photons with energy lower than the bandgap of the top cell can be absorbed in this substrate.

The performance of this solar cell can be modeled analytically following the ambipolar diode equation formulated by Shockley $[1,2]$ generalized to include carrier generation due to light illumination as in Hovel's work [3]. We will call this model along this paper the "SH model". The differential equations of this model, together with the boundary conditions, are given in the Annex. They are second order linear differential equations that can be solved analytically. However, we will not write their solution in this manuscript since we find it not practical. Nowadays, once the problem has been formulated in terms of differential equations as we do in the Annex, programs such as Mathematica or Mapple can solve them at the time they minimize the risk of introducing typing errors. Following this procedure, the model allows calculating, in particular, the current-voltage characteristic of the cell and its internal quantum efficiency. The results, however, lead us to an interesting puzzle discussed in the next sections.

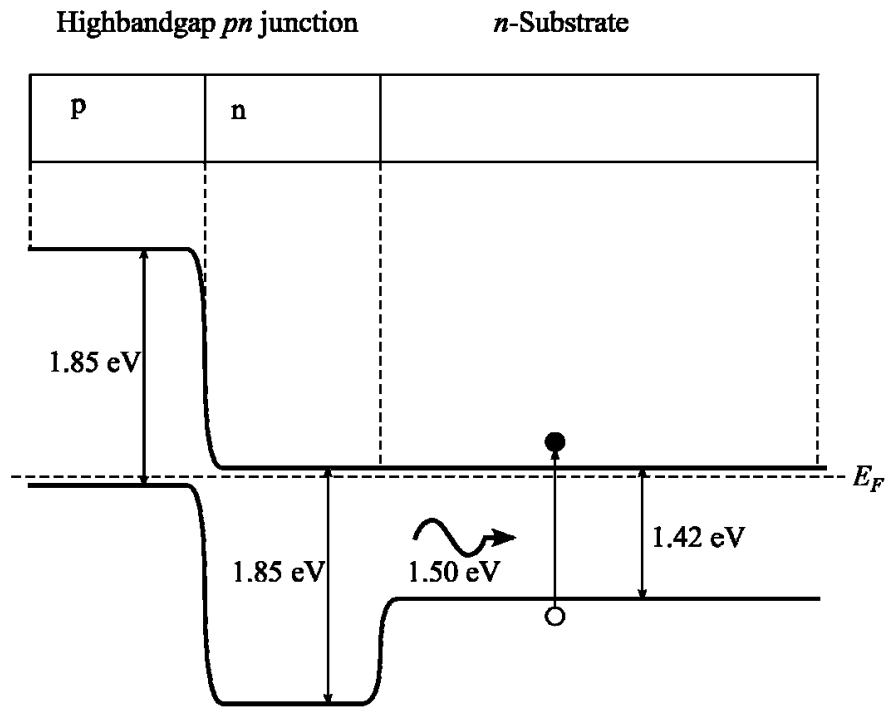

Fig. 1. Structure and bandgap diagram in equilibrium of a high bandgap solar cell grown on a substrate of lower bandgap.

\section{Current Voltage Characteristic}

To illustrate with numbers our puzzle, we will assume that the high bandgap top cell is made of $\mathrm{Al}_{0.3} \mathrm{Ga}_{0.7} \mathrm{As}$ (that has a bandgap of $1.85 \mathrm{eV})$ and the substrate is made of GaAs (1.42 $\mathrm{eV}$ bandgap). The material parameters of the cell structure assumed in our simulations as well as the values for the minority carriers transport and recombination properties are given in Table I. The results, however, are general and not very much dependent on these values as long as they are kept within physically meaningful boundaries. The figures in Table I are given here as an example to facilitate that our results and discussion can be checked by others.

Being dealing with an heterostructure, spikes will usually appear in the bandgap diagram at the AlGaAs/GaAs interface. We will ignore them since we consider that they do not affect our main argument. The reader confused by this fact can either assume that carriers can easily tunnel through these spikes or that we are dealing with hypothetical materials in which their electron affinity has being finely tuned to reproduce the bandgap diagram in Fig. 1. Along this simplifying line, we will also assume the cell illuminated with 
$6000 \mathrm{~K}$ black body radiation. Again, changing the illumination for a more realistic AM1.5 spectrum does not change the validity of our results but would make it more difficult for others to study and reproduce them.

TABLE I

DETAILS OF THE STRUCTURE MODELED AND MINORITY CARRIER VALUES

\begin{tabular}{|l|c|c|c|}
\hline \multicolumn{1}{|c}{ AND MINORITY CARRIER VALUES } \\
\hline Thickness $(\mu \mathrm{m})$ & $\begin{array}{c}\mathrm{p}- \\
\mathrm{Al}_{0.3} \mathrm{Ga}_{0.7} \mathrm{As}\end{array}$ & $\begin{array}{c}\mathrm{n}- \\
\mathrm{Al}_{0.3} \mathrm{Ga}_{0.7} \mathrm{As}\end{array}$ & $\begin{array}{c}\mathrm{n} \text {-GaAs } \\
\text { substrate }\end{array}$ \\
\hline Doping $\left(\mathrm{cm}^{-3}\right)$ & $10^{17}$ & $10^{17}$ & 1 \\
\hline $\begin{array}{l}\text { Minority carrier } \\
\text { Lifetime }(\mathrm{ns})\end{array}$ & 30 & 30 & $10^{17}$ \\
\hline $\begin{array}{l}\text { Mobility } \\
\left(\mathrm{cm}^{2} \mathrm{~V}^{-1} \mathrm{~s}^{-1}\right)\end{array}$ & 1200 & 1200 & 1200 \\
\hline $\begin{array}{l}\text { Surface } \\
\text { recombination }\end{array}$ & $0 \mathrm{~cm} \mathrm{~s}^{-1}$ & - & $0 \mathrm{~cm} \mathrm{~s}^{-1}$ \\
\hline $\begin{array}{l}\text { Intrinsic } \\
\text { concentration }\end{array}$ & $280 \mathrm{~cm}^{-3}$ & $280 \mathrm{~cm}^{-3}$ & $10^{6} \mathrm{~cm}^{-3}$ \\
\hline $\begin{array}{l}\text { Absorption } \\
\text { coefficient }\end{array}$ & $\begin{array}{c}\text { Ref. [4] } \\
\text { with } h=0\end{array}$ & $\begin{array}{c}\text { Ref. }[4] \\
\text { with } h=0\end{array}$ & $\begin{array}{c}\text { Ref. }[4] \\
\text { with } h=0\end{array}$ \\
\hline Intrinsic level & midgap & midgap & midgap \\
\hline
\end{tabular}

With these premises, Fig. 2 plots the current-voltage characteristic of this cell under a light concentration of 1000 suns. Nothing looks harmful at this point yet. We call attention, though, to the fact that the open-circuit voltage of the cell, $1.68 \mathrm{~V}$, multiplied by the electron charge is higher than the bandgap of the substrate $(1.42 \mathrm{eV})$. This is usually considered a trivial result since the substrate is traditionally considered as photovoltaic inactive. For reference, we point out that, at one sun, the resulting open-circuit voltage is 1.502 $\mathrm{V}$ which still is also higher than $1.42 \mathrm{~V}$.

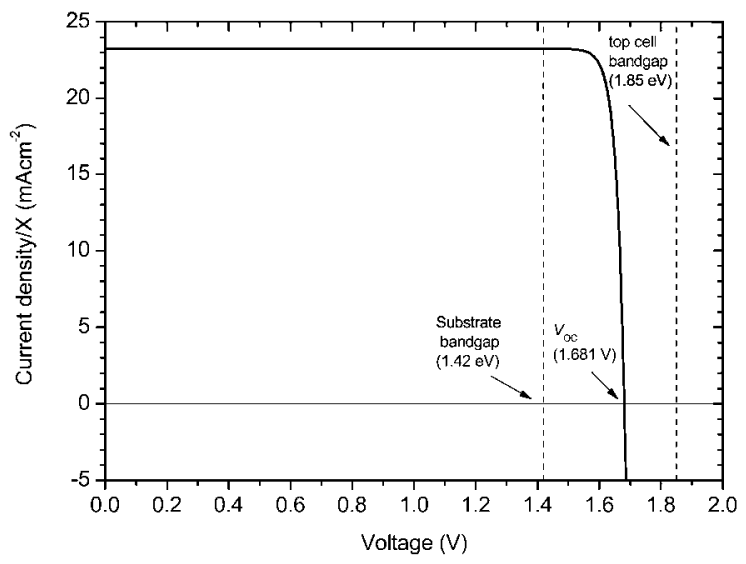

Fig. 2. Current voltage characteristic of the high bandgap solar cell on low bandgap substrate

\section{QUANTUM EFFICIENCY}

However, in our example, we do not make the apriori assumption of a photovoltaic inactive substrate and do include in our model the fact that photons with energy lower than the top cell bandgap can be absorbed in it. In fact, the application of the SH model to calculate the quantum efficiency predicts that it is actually possible to generate photocurrent from these photons. Observe, for example, in the plot in Fig. 3 that, although small, carriers generated by 0.83 $\mu \mathrm{m}(1.5 \mathrm{eV})$ photons can be extracted with quantum efficiency of $6 \times 10^{-6}$. Here is where the puzzle appears: if a $1.5 \mathrm{eV}$ photon can be extracted at a voltage of say $1.6 \mathrm{~V}$ (which apparently is possible given the results in Fig. 2) it means that we are able to extract an energy from it of $1.6 \mathrm{eV}$, which violates the principles of thermodynamics. What is wrong here? Is perhaps the SH model wrong? If it is, we are in serious trouble because it is a fundamental model used for years to study solar cells. Even if it is wrong, what is the solution to the puzzle? Must we perhaps impose ad hoc that the substrate cannot produce photocurrent at all? Must we impose that the open-circuit of the cell is limited to the bandgap of the substrate?

\section{THE SOLUTION}

The solution to the puzzle is the following.

Fortunately, the SH model is consistent also for this structure. The output voltage of the cell in the current-voltage characteristic in Fig. 2 is not limited by the substrate bandgap because the cell is illuminated with a multicolour spectrum (the sun) and most of the photons are absorbed at the top pnjunction. The voltage for the extraction of the carriers generated by these photons is limited by the top pn junction bandgap and not by the bandgap of the substrate.

However, if we illuminate the cell with monochromatic photons that can only be absorbed in the substrate, we find that the voltage is limited by the bandgap of the substrate. This is illustrated by means of the plot in Fig. 4 that shows the current-voltage characteristic of the cell when illuminated with a monochromatic $1.5 \mathrm{eV}$ photon beam at different intensities. Notice the scale of the plot is now $\mu \mathrm{Acm}^{-2}$ and the increasing difficulty in extracting current from the cell as the power of the incident beam is increased. This difficulty is accompanied by the difficulty in increasing the open-circuit voltage of the cell that even remains far from the $1.42 \mathrm{~V}$ limit set by the bandgap of the substrate.

The reason for the bandgap of the substrate limiting the open-circuit voltage relies on the fact that, when illuminating with a $1.5 \mathrm{eV}$ photon beam, the major increase in carrier population over equilibrium takes place at the substrate. Then, the quasi-Fermi level split between electrons and holes reaches its maximum also at the substrate where the split is limited by the substrate bandgap due to the appearance of stimulated emission. In our model we have taken this feature 
into account as follows: if after solving the system of differential equations formulated in the Annex it is found that the split of quasi-Fermi levels at the substrate becomes higher than the bandgap, then the value of the minority carrier lifetime is decreased until the quasi-Fermi level split is kept within the bandgap boundaries (In the example in Fig. 4, for the $10 \mathrm{Wcm}^{-2}$ case this condition implied the reduction of the minority carrier lifetime at the substrate from $30 \mathrm{~ns}$ to $3 \mathrm{~ns}$ )

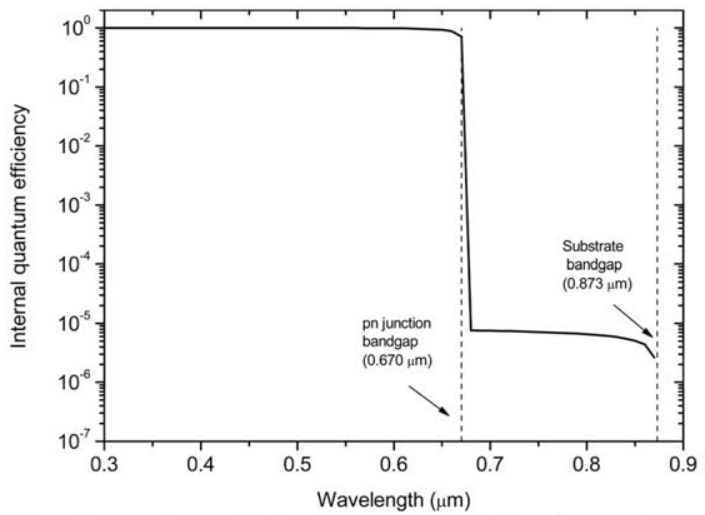

Fig. 3. Internal quantum efficiency of the high bandgap solar cell on low bandgap substrate.

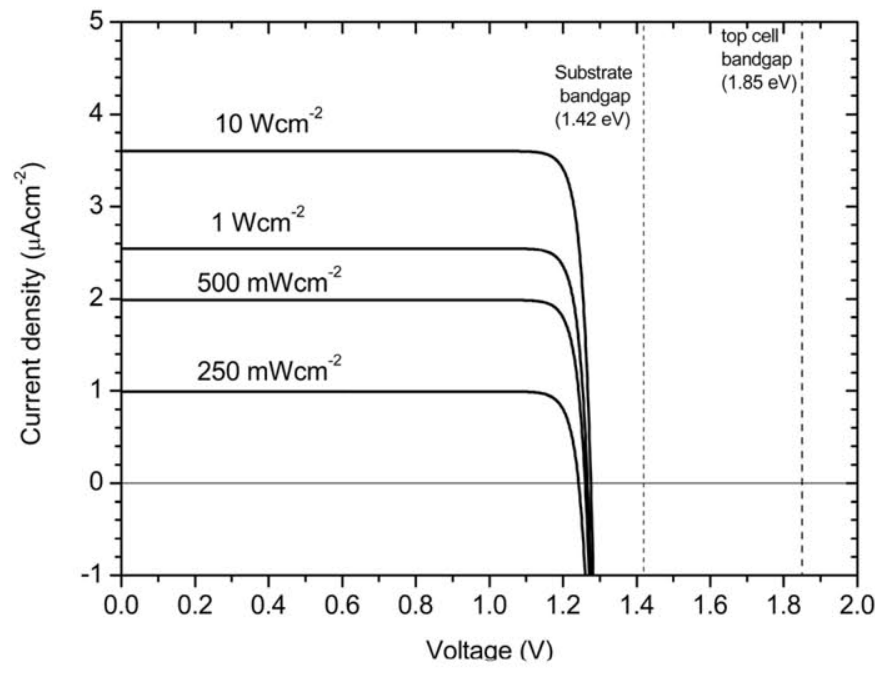

Fig. 4. Current voltage characteristics of the high bandgap solar cell on low bandgap substrate when illuminated with a $1.5 \mathrm{eV}$ monochromatic beam. The power of the beam is indicated in the plot for each characteristic. The photons of this beam can only be absorbed at the substrate.

\section{IMPACT FOR THE INTERMEDIATE BAND SOLAR CELL RESEARCH}

Several lessons can be learnt from the discussion and analysis carried out in the previous paragraphs that are of applicability to the research on intermediate band solar cells
(IBSCs). We assume the reader familiarized with this concept. If not he can read, for example, [5] to learn about the fundamentals and [6] for a review related to the appropriate interpretation of the experimental results before continuing with this section.
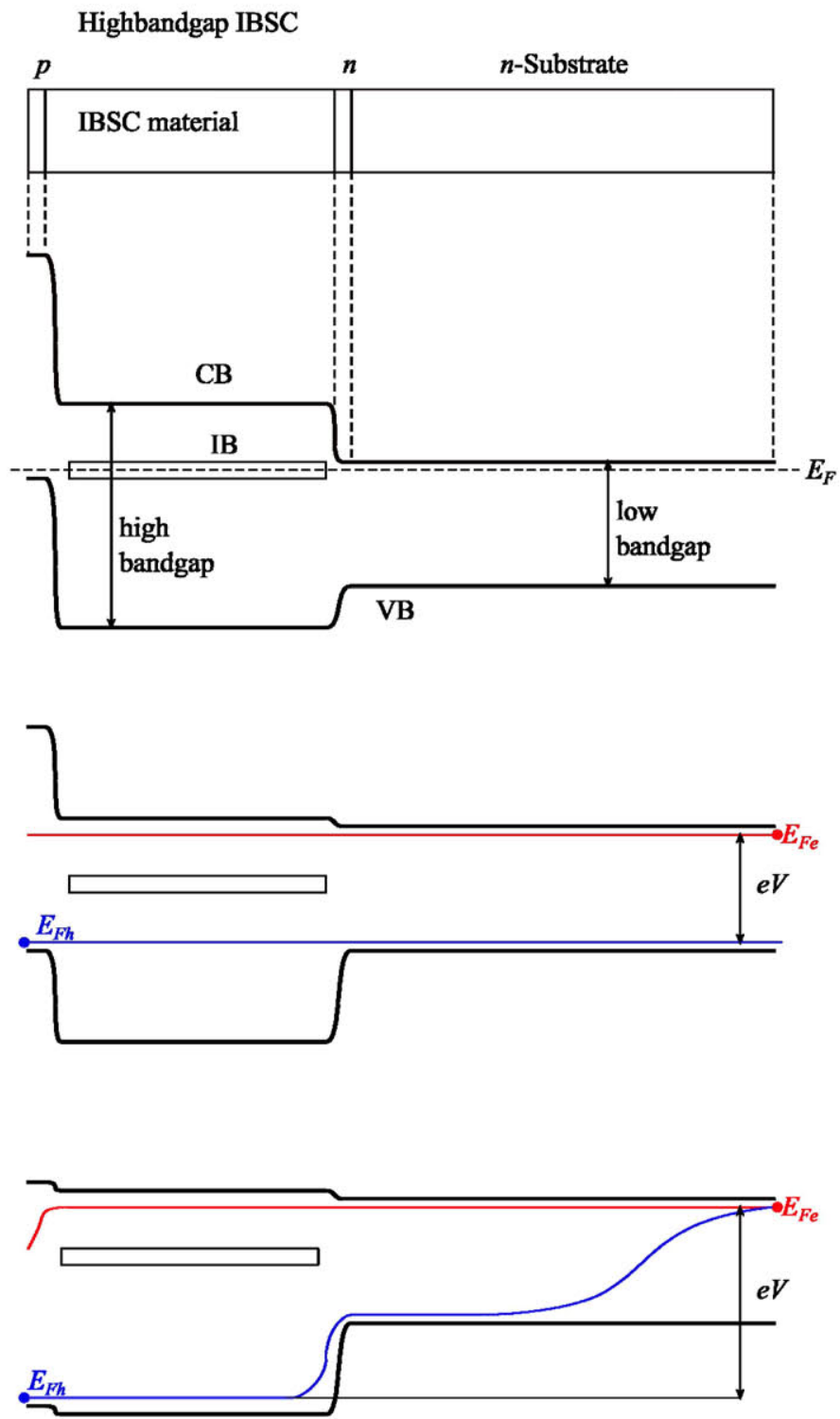

Fig. 5. Top: Example of IBSC structure grown on a low bandgap substrate and approximated bandgap diagram in equilibrium. $E_{F}$ represents the Fermi level. Middle: Bandgap diagram showing electron $\left(E_{F e}\right)$ and hole $\left(E_{F h}\right)$ quasi-Fermi levels under the strict hypothesis of infinite mobility. $\mathrm{eV}$ is the output voltage of the cell multiplied by the electron charge. Bottom: As in the middle plot but allowing for non-infinite mobility.

Let us assume, first, that the top high bandgap cell in Fig. 1 is substituted by an IBSC structure as the one described, for example in [7]. This would lead us to the structure sketched in Fig. 5 (top). We recall that the total bandgap of an ideal IBSC should also be high, in the range of $2 \mathrm{eV}$. It is probable 
that they are grown on substrates of lower bandgap, and therefore, knowing whether the use of such substrates imposes a limitation for the cell performance or not becomes a relevant topic.

In this sense, it might be feared that the growth of this IBSC structure on a substrate with lower bandgap might result in the open-circuit of the IBSC limited by the bandgap of this substrate (say to $1.4 \mathrm{~V}$ if the bandgap is $1.4 \mathrm{eV}$ ) and not by the total bandgap of the IBSC ( $2 \mathrm{~V}$ in this example). This fear has its roots on the assumption of infinite carrier mobility that would lead us to an electron and hole quasi-Fermi level distributed along the structure as sketched in Fig. 5 (middle). Since the electron and hole quasi-Fermi level split cannot exceed the substrate bandgap, then the output voltage of the cell becomes in this case limited by the bandgap of the substrate.

However, the extrapolation of the analysis carried out in the previous sections reveals that this has not to be the case when the carrier mobility is finite and therefore, the lower bandgap of the substrate might not be a limiting factor for the output voltage of the cell. This would be explained by a quasi-Fermi level distribution as the one sketched in Fig. 5 (bottom). Notice that the hypothesis of finite mobility leads to ohmic losses. However, notice also that the regions where the gradient of quasi-Fermi level become more apparent are also the regions where the carrier related to the relevant quasiFermi level becomes minority so that at the end, the ohmic losses might not be as high as the plot in Fig. 5 (bottom) might induce us to think at first sight. This consideration makes us also to think of the solar cell efficiency not only in terms of shortcircuit-current and open-circuit voltage tradeoffs, but also in terms of fill factor. Hence, in the same way that trade-offs of the kind "a voltage loss can be compensated by a shortcircuit gain", trade-offs of the kind "a reduction of fill factor can be compensated by a voltage gain" should also be considered in complex solar cell structures.

Finally, notice that if an IBSC structure based on a high bandgap semiconductor is grown on a lower bandgap semiconductor, there might be contribution from the substrate to the quantum efficiency of the device since photons reaching the substrate might be revealed in a quantum efficiency measurement. A sufficiently sensitive quantum efficiency setup might still be able to measure this photocurrent, even if a BSF layer is inserted in-between both regions, and, perhaps, lead to a misinterpretation of the results in the sense that a current originated at the substrate might be wrongly attributed to the IBSC.

\section{CONCLUSIONS}

When a high bandgap cell structure is grown on a low bandgap semiconductor, the photons absorbed by the substrate can contribute to the cell photocurrent. This could lead us to the temptation of believing that we have a device capable of extracting at high voltage electrons generated by means of the absorption of low energy photons (the ones absorbed by the substrate). However, a careful analysis reveals that these electrons cannot be extracted at the high voltage that the top pn junction can sustain but that, instead, their electrochemical potential is also limited by the substrate bandgap. In case a high bandgap IBSC is grown on a low bandgap semiconductor, care must be exercised in order to distinguish which part of the below bandgap photogenerated current is contributed by the IB and which part is eventually contributed from the substrate. On the other hand, an IBSC grown on a low bandgap semiconductor should not necessarily see its output voltage limited by the substrate bandgap when illuminated with polychromatic light.

\section{ACKNOWLEDGMENTS}

This work has been supported by European Commission through the NGCPV Project (Grant Number 283798). EA acknowledges a Marie Curie Fellowship from the European Commission (Project Siracusa, Grant PIEF-GA-2011302489). IR acknowledges UPM Grant (call RR01/2009). EL acknowledges the "Ayudas para la Formación de Personal Investigador (FPI) of the Spanish Government (EEBB-BES2010-037659).

\section{ANNEX I}

Here we give the differential equations that model the solar cell structure in Fig. 1 together with its boundary conditions. The solution to these equations is analytical and is the one used in the calculations shown in this paper. The equations assume implicitly a low injection regime.

\footnotetext{
DEFINITIONS

$\Delta y_{x} ;$ minority carrier excess in region 1

region 1: emitter top cell

region 2: base top cell

region 3 : substrate

$n_{i, x}$ : intrinsic concentration in region $x$

$\alpha_{x}$; absorption coefficient in region $x$

$D_{x}^{x} ;$ minority carrier diffusion constant in region $x$

$L_{x}^{x}$; minority carrier diffusion length in region $x$

$F_{0}$; incident photon flux at the surface (photons $\mathrm{cm}^{-2} \mathrm{~s}^{-1} \mathrm{~mm}^{-1}$ )

$W_{x}$; Thickness of region $x$

$S_{1} ;$ Front surface recombination

$S_{3}$; Back surface recombination

$V$; Voltage at cell terminals

$N_{x}$; doping at region $x$

$\mu_{23}$; quasi-Fermi level split at the interface between top cell and substrate
} 
[4] A. Martí and G. L. Araújo, "Gallium Arsenide material parameter models for solar cell simulation,"

\section{REGION 1}

$$
\begin{aligned}
& \frac{d^{2} \Delta y_{1}}{d x^{2}}=\frac{\Delta y_{1}}{L_{1}}-F_{0} \alpha_{1} \exp \left(-\alpha_{1}\left(x-W_{1}\right)\right) \\
& D_{1} \frac{d \Delta y_{1}}{d x} \Delta y_{1}\left(-W_{1}\right)=S_{1} \Delta y_{1}\left(-W_{1}\right) \\
& \Delta y_{1}(0)=\frac{n_{i, 1}}{N_{1}}\left[\exp \left(\frac{e V}{k T}\right)-1\right]
\end{aligned}
$$

\section{REGION 2}

$$
\begin{aligned}
\frac{d^{2} \Delta y_{2}}{d x^{2}} & =\frac{\Delta y_{2}}{L_{2}}-F_{0} \exp \left(-\alpha_{1} W_{1}\right) \alpha_{2} \exp \left(-\alpha_{2} x\right) \\
\Delta y_{2}(0) & =\frac{n_{i, 2}}{N_{2}}\left[\exp \left(\frac{e V}{k T}\right)-1\right] \\
\Delta y_{2}\left(W_{2}\right) & =\frac{n_{i, 2}}{N_{2}}\left[\exp \left(\frac{\mu_{23}}{k T}\right)-1\right]
\end{aligned}
$$

\section{REGION 3}

$$
\begin{aligned}
& \frac{d^{2} \Delta y_{3}}{d x^{2}}=\frac{\Delta y_{3}}{L_{3}}-F_{0} \exp \left(-\alpha_{1} W_{1}\right) \exp \left(-\alpha_{2} W_{2}\right) \alpha_{3} \exp \left(-\alpha_{3} x\right) \\
& \Delta y_{3}(0)=\frac{n_{i, 3}}{N_{3}}\left[\exp \left(\frac{e \mu_{23}}{k T}\right)-1\right] \\
& -D_{3} \frac{d \Delta y_{3}}{d x} \Delta y_{3}\left(W_{3}\right)=S_{3} \Delta y_{3}\left(W_{3}\right) \\
& D_{3} \frac{d \Delta y_{3}}{d x} \Delta y_{3}(0)=D_{2} \frac{d \Delta y_{2}}{d x} \Delta y_{2}\left(W_{2}\right)
\end{aligned}
$$

\section{QUANTUM EFFICIENCY (for photons with energy $E$ )}

$$
Q E(E)=\left[D_{1} \frac{d \Delta y_{1}}{d x}(0)-D_{2} \frac{d \Delta y_{2}}{d x}(0)\right] / F_{0} \quad(\text { with } V=0)
$$

DARK current-voltage characteristic $\left(F_{0}=0\right)$

$$
J_{D}(V)=D_{1} \frac{d \Delta y_{1}}{d x}(0)-D_{2} \frac{d \Delta y_{2}}{d x}(0) \quad\left(\text { with } F_{0}=0\right)
$$

\section{CURRENT-VOLTAGE characteristic}

$$
J(V)=\int_{E} F_{0} Q E(E) d E-J_{D}(V)
$$

Proc. of the 11th E.C Photovoltaic Solar Energy Conference, pp. 291--294, 1992.

A. Luque and A. Martí, "Increasing the efficiency of ideal solar cells by photon induced transitions at intermediate levels," Physical Review Letters, vol. 78, pp. 5014-5017, 1997.

A. Marti, E. Antolin, P. G. Linares, and A. Luque, "Understanding experimental characterization of intermediate band solar cells," Journal of Materials Chemistry, 2012.

A. Luque and A. Martí, "A metallic intermediate band high efficiency solar cell," Progress in Photovoltaics: Res. Appl., vol. 9, pp. 73-86, 2001.

\section{REFERENCES}

[1] W. Shockley, "The Theory of p-n junctions in Semiconductors and p-n Junction Transistors," Bell Syst. Tech. J., vol. 28, pp. 435-489, 1949.

[2] M. Kelvy, Fisica del estado sólido y semiconductores. Lima: Limusa, 1989.

[3] H. J. Hovel, Solar Cells vol. 11. New York: Academic Press, 1975. 